806

COMUNICAÇÃO CIENTÍFICA

\title{
PRODUTIVIDADE E QUALIDADE DA 'CABERNET SAUVIGNON' SUSTENTADA EM ESPALDEIRA E MANJEDOURA NA FORMA DE Y ${ }^{1}$
}

\author{
MÁRIO JOSÉ PEDRO JÚNIOR², JOSÉ LUIZ HERNANDES 3 , \\ GABRIEL CONSTANTINO BLAIN ${ }^{4}$, LUDMILA BARDIN-CAMPAROTTO ${ }^{5}$
}

RESUMO - A uva 'Cabernet Sauvignon' utilizada para elaboração de vinho tem tido seu plantio incrementado na região vitivinícola de São Roque (SP), sendo o sistema de sustentação mais usual a espaldeira. Recentemente, foi introduzido o sistema de sustentação em manjedoura na forma de Y, portanto foi realizado experimento objetivando caracterizar a produção e as características químicas do mosto da 'Cabernet Sauvignon' sustentada em espaldeira e em Y. As avaliações foram feitas durante os anos agrícolas: 2010/2011, 2011/2012 e 2012/2013. As videiras sustentadas em Y apresentaram maiores médias de massa dos cachos e de produtividade quando comparadas às sustentadas em espaldeira. As médias de produtividade estimada variaram entre 9,89 e 13,64 t ha- ${ }^{-1}$ para a espaldeira e entre 13,34 e 17,43 tha $\mathrm{tara}^{-1}$ a manjedoura na forma de Y. Em relação às características químicas do mosto (teor de sólidos solúveis, pH e acidez titulável), não foram observadas diferenças estatísticas entre os sistemas de sustentação das videiras. As médias do teor de sólidos solúveis variaram entre 17,3 e $19,3^{\circ}$ Brix, e a acidez titulável total, entre 98 e 143 meq.. $\mathrm{L}^{-1}$ para os diferentes anos agrícolas avaliados.

Termos de indexação: uva para vinho, Vitis vinifera, sistema de sustentação.

\section{YIELD AND QUALITY OF 'CABERNET SAUVIGNON' TRAINED UNDER VERTICAL SHOOT POSITION AND Y SHAPPED TRELLIS SYSTEMS}

\begin{abstract}
The grapevine 'Cabernet Sauvignon' used for wine purposes is increasing its cultivation in the region of São Roque (State of São Paulo, Brazil) being the vertical shoot position (VSP) system the most usual among the growers. Recently it was introduced the $\mathrm{Y}$ training system, therefore a field trial was carried out to characterize yield and chemical characteristics of the must of 'Cabernet Sauvignon' grown under different training systems. Evaluations were made during the following growing seasons: 2010/11, 2011/12 and 2012/13. The grapevines trellised on the Y shape showed higher cluster weight and yield when compared to the VSP system. The obtained values of yield varied from 9.89 and $13.64 \mathrm{t} \mathrm{ha}^{-1}$ for the VSP system and from 13.34 and $17.43 \mathrm{tha}^{-1}$ for the $\mathrm{Y}$ shaped system. Regarding the chemical characteristics of the must (soluble solids, $\mathrm{pH}$ and titratable acidity) it was not found statistical differences when comparing the training systems. The obtained values of total soluble solids content varied from 17.3 and $19.3^{\circ}$ Brix and titratable acidity from 98 and 143 meq. $L^{-1}$ for different growing seasons evaluated.
\end{abstract}

Index terms: grapevine, soluble solids, trellising system, training system.

\footnotetext{
${ }^{1}$ (Trabalho 140-14). Recebido em: 2-04-2014. Aceito para pubicação em: 29-09-2014.

${ }^{2}$ Eng. Agr. Dr., PqC Centro de Ecofisiologia e Biofísica, IAC, Caixa Postal 28, Campinas-SP, Brasil, e-mail: mpedro@iac.sp.gov.br. Bolsista CNPq

${ }^{3}$ Biólogo MS., PqC Centro de Fruticultura, IAC, Jundiaí, SP, Brasil, e-mail: jlhernandes@iac.sp.gov.br

${ }^{4}$ Eng. Agrícola Dr., PqC Centro de Ecofisiologia e Biofísica, IAC, Caixa Postal 28, Campinas-SP, Brasil, e-mail: gabriel@iac.sp.gov.br ${ }^{5}$ Eng. Amb. Dra., Pós-Doutoranda, Centro de Ecofisiologia e Biofísica, IAC, Caixa Postal 28, Campinas-SP, Brasil, e-mail: ludmila bardin@yahoo.com.br. Bolsista FAPESP.
} 
A viticultura da região de São Roque (SP), tradicionalmente, está baseada em cultivares rústicas de uvas para vinho e na utilização do sistema em espaldeira para sustentação das videiras. Recentemente, devido ao incremento do turismo, a busca por vinhos de melhor qualidade aumentou, direcionando o produtor para o plantio de uvas viníferas para obtenção de vinhos finos.

Nesse contexto, o viticultor teve sua atenção voltada para a 'Cabernet Sauvignon', de origem francesa e de uso já consolidado na região Sul do País (CAMARGO et al., 2011). Esta cultivar de uva foi avaliada para elaboração de vinho tinto, em Bento Gonçalves (RS), em relação às características agronômicas e enológicas por Rizzon e Miele (2002). Esses autores relataram valores médios de cinco safras, informando que os cachos tinham massa de $149 \mathrm{~g}$, e o mosto apresentou teor de sólidos solúveis de 20,6 ${ }^{\circ}$ Brix e acidez titulável de 120 meq. $\mathrm{L}^{-1}$. Ainda, na região Sul do País, Santos et al. (2010), avaliando o efeito do raleio de cachos na qualidade da 'Cabernet Sauvignon' conduzida em latada, verificaram valores de teor de sólidos solúveis, durante três safras, variando entre 17,6 e $23,6{ }^{\circ}$ Brix. Na região norte do Paraná, Santos et al. (2007) obtiveram valores inferiores de teor de sólidos solúveis $\left(14,2^{\circ} \mathrm{Brix}\right)$ com produtividade em torno de 15,9 $\mathrm{t} \mathrm{ha}^{-1} \mathrm{em}$ videiras conduzidas em latada. Em Santa Catarina, Brighenti et al. (2011), em região de altitude, relataram produtividade entre 7,2 e 13,1 tha ${ }^{-1}$, e teor de sólidos solúveis na faixa de 20 a $22^{\circ}$ Brix para a 'Cabernet Sauvignon' enxertada sobre diferentes porta-enxertos.

Em Minas Gerais, na região sul cafeeira, Mota et al. (2010) avaliaram o potencial de maturação de diferentes cultivares de uvas para vinho fino em ciclos de verão e de inverno. Esses autores relataram que a 'Cabernet Sauvignon', durante a safra de verão, mostrou teor de sólidos solúveis em torno de $14^{\circ}$ Brix e acidez total de 124 meq. $\mathrm{L}^{-1}$.

No Estado de São Paulo, na região de Jundiaí, Ferreira et al. (2004) obtiveram produções de até 1,5 kg.planta ${ }^{-1}$ para a 'Cabernet Sauvignon' sustentada no sistema em espaldeira a céu aberto. Também, Orlando et al. (2008) avaliaram a cultivar na mesma região, verificando produções de 2,06 kg.planta-1 para o porta-enxertos IAC 571-6 'Jundiaí'. Enquanto, Regina et al. (2011), na região nordeste do Estado de São Paulo (município de Espírito Santo do Pinhal), obtiveram elevado teor de sólidos solúveis, em torno de $25^{\circ}$ Brix, para a 'Cabernet Sauvignon' colhida entre os meses de maio e junho.

Esses resultados promissores de produtividade e, principalmente, de parâmetros enológicos levaram os produtores de São Roque a utilizar essa cultivar sustentada em espaldeira. Recentemente, foi introduzido o sistema de condução em manjedoura na forma de $\mathrm{Y}$, que se tornou nova opção para manejo dos vinhedos (SCHUCK, 2004; FALCÃO et al., 2008; CAMARGO et al., 2011; HERNANDES et al., 2013) devido às vantagens relativas à maior exposição das folhas ao sol, por propiciar maior ventilação dos cachos, permitir realização de poda mista e facilitar a locomoção do produtor no vinhedo, apesar do custo de implantação mais elevado. Em experimento realizado por Falcão et al. (2008), foi feita a comparação da maturação da 'Cabernet Sauvignon', na região de São Joaquim (SC), sustentada nos sistemas em espaldeira e em Y. Os autores obtiveram pequena diferença entre os valores de teor de sólidos solúveis (entre 22 e $24^{\circ} \mathrm{Brix}$ ) e de acidez titulável total (entre 0,68 e 0,75 g. $100 \mathrm{~mL}^{-1}$ ) em função da influência marcante da ocorrência de chuvas durante o período de maturação.

Portanto, levando-se em consideração os resultados relativos à produtividade e ao potencial para acúmulo de açúcares descritos nos estudos anteriormente citados, objetivou-se neste trabalho determinar as características fitotécnicas e químicas do mosto da 'Cabernet Sauvignon' sustentada em espaldeira e em manjedoura, na forma de Y, na região vitícola de São Roque (SP).

$\mathrm{O}$ ensaio foi conduzido em vinhedo da Vinícola Góes, localizado no município de São Roque (SP) e situado a $850 \mathrm{~m}$ de altitude, latitude $47^{\circ} 08^{\prime} \mathrm{W}$ e longitude $23^{\circ} 32^{\prime} \mathrm{S}$. O clima segundo a classificação de Köeppen é Cfb, e durante a fase de maturação das uvas, que ocorre entre fevereiro e março, o valor médio de temperatura mensal é 22,5 ${ }^{\circ} \mathrm{C}$, com total médio de precipitação pluvial próximo de $425 \mathrm{~mm}$.

Foram utilizadas videiras (Vitis vinifera $\mathrm{L}$.) 'Cabernet Sauvignon' enxertadas sobre Paulsen 1103, sustentadas no sistema em espaldeira, com espaçamento de $1,2 \times 2,7 \mathrm{~m}$, com densidade aproximada de 3.100 plantas.ha $^{-1}$, e em manjedoura na forma de $\mathrm{Y}$, com espaçamento de $1,5 \times 3,0 \mathrm{~m}$, com densidade aproximada de 2.220 plantas.ha ${ }^{-1}$. Os tratos culturais (adubação, poda verde) e fitossanitários para controle das doenças foram feitos de acordo com a recomendação técnica para a região. Foram realizadas podas curtas no sistema em espaldeira e podas mistas no sistema em manjedoura, na forma de Y, durante os anos agrícolas de 2010/2011; 2011/2012 e 2012/2013. As datas de poda foram: 15 9-10; 22-9-11 e 20-09-12 e as colheitas em: 10/03/11; 27/03/12 e 25/03/13.

O delineamento experimental foi o 
inteiramente casualizado, sendo feita na época de colheita, a contagem em 30 plantas (repetições), do número de cachos, sendo coletados 30 cachos aleatoriamente, para a determinação da massa do cacho por pesagem,em balança de precisão de 0,1 g. A produção por planta foi estimada pelo número e massa média dos cachos. Para a avaliação da qualidade do mosto, no momento da colheita, foram coletadas aleatoriamente 120 bagas em cada um dos sistemas de condução, divididas depois em quatro amostras de 30 bagas. Foram avaliados: teor de sólidos solúveis (TSS), expresso em ${ }^{\circ}$ Brix, por meio de refratômetro óptico manual, com escala de 0-32; $\mathrm{pH}$, utilizando-se pHmetro de bancada; acidez titulável (AT) em meq. $\mathrm{L}^{-1}$, realizada por titulação do mosto com solução padronizada de $\mathrm{NaOH} 0,1 \mathrm{~N}$, tendo sido ajustado para $\mathrm{pH}=8,2$ como ponto final da titulação. Os valores médios das características fitotécnicas das videiras (produção, produtividade estimada, número de cachos); do cacho (massa) e características químicas do mosto (TSS, pH e acidez total) foram submetidos à análise de variância e comparados pelo teste " $t$ ".

Variáveis fitotécnicas - os valores de massa dos cachos variaram entre 122,2 e 175,9 g durante o período experimental. Esses valores foram superiores aos relatados por Mota et al. (2008), também para a 'Cabernet Sauvignon' sustentada em Y, sobre o porta-enxerto Paulsen 1103, cultivada em Caxias do Sul, e da mesma ordem de grandeza dos obtidos por Rizzon e Miele (2002), em Bento Gonçalves. Foi observada diferença estatística entre os sistemas de sustentação, tendo sido observados cachos de maior massa para a manjedoura na forma de Y, em comparação ao espaldeira (Tabela 1). Em relação ao número de cachos, observou-se que as videiras do sistema em Y produziram maior número de cachos quando comparado ao sistema espaldeira. Para o sistema em espaldeira, o número de cachos variou entre 26 e 32 por planta, enquanto para o sistema em $\mathrm{Y}$, os valores foram entre 41 e 47 cachos por planta. Essa diferença pode ser explicada pelo fato de ter sido utilizada poda curta no sistema em espaldeira e poda mista no sistema em Y, que pelo maior número de gemas favorece a produção de maior número de cachos nos ramos em que se pratica a poda longa. $\mathrm{O}$ número de cachos obtidos neste experimento foi superior aos relatados por Mota et al. (2008), que obtiveram 24 cachos por planta, e também por Regina et al. (2011), no nordeste de São Paulo (5 cachos por planta).

No que diz respeito à produção e à produtividade estimada, verificou-se diferença estatística entre os sistemas de sustentação avaliados. O sistema em Y apresentou, nos três anos agrícolas avaliados, médias superiores à espaldeira. Essa diferença entre sistemas de sustentação pode ser explicada pela maior massa do cacho e número de cachos no sistema em Y. As médias de produtividade do sistema em Y (entre 13,3 e 17,4 t ha ${ }^{-1}$ ) são superiores aos resultados relatados por Mota et al. (2010) para Minas Gerais e semelhantes aos obtidos por Santos et al. (2007) para a região norte do Paraná. Apesar da maior produtividade observada no sistema de condução em Y, em comparação à espaldeira, deve ser ressaltado seu custo mais elevado na implantação do vinhedo (HERNANDES et al., 2013).

Variáveis químicas do mosto - as variáveis químicas do mosto avaliadas na época da colheita (TSS, pH e AT) não diferiram estatisticamente entre si, pelo teste " $t$ ", ao nível de $5 \%$, na comparação dos sistemas de sustentação dentro do mesmo ano agrícola. A análise foi feita apenas dentro do mesmo ano agrícola, pois existe variação entre anos agrícolas das variáveis químicas do mosto normalmente influenciadas pelas condições climáticas, principalmente pela ocorrência de chuvas (FALCÃO et al., 2008; SANTOS et al., 2010),fato que dificulta a comparação entre os anos agrícolas. As médias obtidas de TSS (Tabela 1) variaram entre 19,1 e $19,3{ }^{\circ}$ Brix nos anos agrícolas de 2011/2012 e 2012/2013, enquanto em 2010/2011 foram inferiores (17,3 a $17,9^{\circ}$ Brix). As médias mais baixas obtidas neste experimento foram semelhantes às relatados por Rizzon e Miele (2002) para a 'Cabernet Sauvignon' cultivada em Bento Gonçalves (RS) e por Orlando et al. (2008) em Jundiaí (SP). Porém foram inferiores às relatados por Regina et al. (2011) para a região nordeste de São Paulo para videiras submetidas ao regime de dupla poda para obtenção de colheita no inverno, com menor ocorrência de chuvas na maturação e na colheita (cerca de $25^{\circ}$ Brix).

As médias de $\mathrm{pH}$ variaram entre 3,17 e 3,31, consideradas compatíveis para fins de elaboração de vinho, que segundo Rizzon e Miele (2002) não devem ultrapassar o valor $\mathrm{pH}=3,3$. A acidez total apresentou médias mais elevadas nos anos agrícolas de 2010/2011 e 2012/2013, em torno de 133 a 143 meq. $\mathrm{L}^{-1}$, sendo comparáveis às relatados por Rizzon e Miele (2002) para a região Sul do País e Orlando et al. (2008) para São Paulo. Durante o ano agrícola de 2011/2012, as médias obtidas foram inferiores, variando entre 98 e 102 meq.L ${ }^{-1}$ para os diferentes sistemas de sustentação.

A comparação entre sistemas de sustentação para a 'Cabernet Sauvignon' permitiu concluir que: 
a) em relação às variáveis fitotécnicas, as videiras no sistema de sustentação em manjedoura na forma de Y, em comparação ao de espaldeira, apresentam maiores médias de massa dos cachos, número de cachos, produção por planta e produtividade estimada; b) com respeito às variáveis químicas do mosto, TSS, pH e AT, não foi observada diferença estatística entre os sistemas de sustentação.

TABELA 1-Variáveis fitotécnicas das plantas e químicas do mosto, da cultivar de uva para vinho Cabernet Sauvignon, para diferentes sistemas de sustentação, durante os anos agrícolas de 2010/2011 a 2012/2013, em São Roque (SP).

\begin{tabular}{|c|c|c|c|c|c|c|c|c|}
\hline \multirow[b]{2}{*}{ Ano agrícola } & \multirow[b]{2}{*}{$\begin{array}{l}\text { Sistema de } \\
\text { sustentação }\end{array}$} & \multicolumn{4}{|c|}{ Variáveis fitotécnicas } & \multicolumn{3}{|c|}{$\begin{array}{c}\text { Variáveis químicas } \\
\text { do mosto }\end{array}$} \\
\hline & & $\begin{array}{l}\text { Massa do } \\
\text { cacho }(\mathrm{g})\end{array}$ & $\begin{array}{c}\text { Número de } \\
\text { cachos } \\
\text { por planta }\end{array}$ & $\begin{array}{c}\text { Produção } \\
\left(\text { kg.planta }{ }^{-1}\right)\end{array}$ & $\begin{array}{l}\text { Produtividade } \\
\text { estimada }\left(\mathrm{t} \mathrm{ha}^{-1}\right)\end{array}$ & $\begin{array}{c}\text { TSS } \\
\left({ }^{\circ} \text { Brix }\right)\end{array}$ & $\mathrm{pH}$ & $\begin{array}{c}\mathrm{AT} \\
\left(\mathrm{meq} \cdot \mathrm{L}^{-1}\right)\end{array}$ \\
\hline \multirow{3}{*}{$2010 / 2011$} & Espaldeira & $141,86 \mathrm{~b}$ & $26,88 \mathrm{~b}$ & $3,81 \mathrm{~b}$ & $11,77 \mathrm{~b}$ & $17,92 \mathrm{a}$ & $3,22 \mathrm{a}$ & $138,04 \mathrm{a}$ \\
\hline & $\mathrm{Y}$ & $175,91 \mathrm{a}$ & $44,94 \mathrm{a}$ & $7,85 \mathrm{a}$ & $17,43 \mathrm{a}$ & $17,31 \mathrm{a}$ & $3,23 \mathrm{a}$ & $133,11 \mathrm{a}$ \\
\hline & $\mathrm{dms}$ & 23,38 & 2,29 & 0,96 & 2,19 & 0,60 & 0,04 & 6,78 \\
\hline \multirow{3}{*}{$2011 / 2012$} & Espaldeira & $138,10 \mathrm{~b}$ & $32,27 \mathrm{~b}$ & $4,45 \mathrm{~b}$ & $13,64 \mathrm{~b}$ & $19,21 \mathrm{a}$ & $3,24 \mathrm{a}$ & $102,52 \mathrm{a}$ \\
\hline & $\mathrm{Y}$ & $150,20 \mathrm{a}$ & $46,93 \mathrm{a}$ & $7,05 \mathrm{a}$ & $15,66 \mathrm{a}$ & $19,05 \mathrm{a}$ & $3,31 \mathrm{a}$ & $98,53 \mathrm{a}$ \\
\hline & dms & 10,42 & 2,73 & 0,38 & 1,36 & 0,42 & 0,10 & 5,19 \\
\hline \multirow{3}{*}{$2012 / 2013$} & Espaldeira & $122,27 \mathrm{~b}$ & $26,32 \mathrm{~b}$ & $3,20 \mathrm{~b}$ & $9,89 \mathrm{~b}$ & $19,07 \mathrm{a}$ & $3,19 \mathrm{a}$ & $138,52 \mathrm{a}$ \\
\hline & $\mathrm{Y}$ & $146,26 \mathrm{a}$ & $41,10 \mathrm{a}$ & $6,01 \mathrm{a}$ & $13,34 \mathrm{a}$ & $19,32 \mathrm{a}$ & $3,17 \mathrm{a}$ & $142,51 \mathrm{a}$ \\
\hline & dms & 16,26 & 2,69 & 0,63 & 1,62 & 0,28 & 0,09 & 6,25 \\
\hline
\end{tabular}

Médias seguidas da mesma letra nas colunas, dentro do mesmo ano agrícola, não diferem entre si, pelo teste t, ao nível de 5\%. TSS = teor de sólidos solúveis; AT = acidez titulável.

\section{AGRADECIMENTOS}

Os autores agradecem à Vinícola Góes localizada no município de São Roque (SP), pelas facilidades oferecidas durante o desenvolvimento deste trabalho.

\section{REFERENCIAS}

BRIGHENTI, A.F.; RUFATO, L.; KRETZCHMAR, A.A.; SCHLEMPER,C. Desempenho vitivinícola da Cabernet Sauvignon sobre diferentes porta-enxertos em região de altitude de Santa Catarina. Revista Brasileira de Fruticultura, Jaboticabal, v.33, n.1, p.96-102, 2011.

CAMARGO, U.A.; TONIETTO, J.; HOFFMANN, A. Progressos na viticultura brasileira. Revista Brasileira de Fruticultura, Jaboticabal, E. p.144149, out. 2011. Volume especial.
HERNANDES, J.L.; PEDRO JÚNIOR, M.J.; BLAIN, G.C.; ROLIM, G.S. Comportamento produtivo da videira 'Niagara Rosada' em diferentes sistemas de condução, com e sem cobertura plástica, durante as safras de inverno e de verão. Revista Brasileira de Fruticultura, Jaboticabal, v.35, n.1, p.123-130, 2013.

FALCÃO, L.D.; CHAVES, E.S.; BURIN, V.M.; FALCÃO, A.P.; GRIS, E.F.; BONIN, V.; BORDIGNON-LUIZ, M.T. Maturity of Cabernet Sauvignon berries from grapevines grown with two different training systems in a new grape growing region in Brazil. Ciencia e Investigatión Agraria, Santiago, v.35, n.3, p. 271-282, 2008.

FERREIRA, M.A.; PEDRO JÚNIOR, M.J.; SANTOS, A.O.; HERNANDES, J.L. Modificação parcial do ambiente de cultivo da videira 'Cabernet Sauvignon' sobre diferentes porta-enxertos: efeito sobre a produção e teor de sólidos solúveis. Bragantia, Campinas, v.63, n.3, p.439-445, 2004. 
MOTA, C.S.; AMARANTE, C.V.T.; SANTOS, H.P.; ZANARDI, O.Z. Comportamento vegetativo e produtivo de videiras 'Cabernet Sauvignon' cultivadas sob cobertura plástica. Revista Brasileira de Fruticultura, Jaboticabal, v.30, n.1, p.148-153. 2008.

MOTA, R.S.; SILVA, C.P.C.; FAVERO, A.C.; PURGATTO, E.; SHIGA, T.M.; REGINA, M.A. Composição físico-química de uvas para vinho fino em ciclos de verão e inverno. Revista Brasileira de Fruticultura, Jaboticabal, v.32, n.4, p.1.127-1.137, 2010.

ORLANDO, T.G.S.; PEDRO JÚNIOR, M.J.; SANTOS, A.O.; HERNANDES, J.L. Comportamento das cultivares Cabernet Sauvignon e Syrah em diferentes porta- enxertos. Ciência e Agrotecnologia, Lavras, v.32, n.3, p.749-755, 2008.

REGINA, M.A.; MOTA, R.V.; FAVERO, A.C.; SHIGA, T.M.; SILVA, L.H.J.; SOUZA, W.C.; NOVELLI, F.A.D.; SOUZA, C.R. Caracterização físico-química de uvas viníferas cultivadas em dupla-poda no nordeste do Estado de São Paulo. Revista Brasileira de Viticultura e Enologia, Bento Gonçalves, v.3, n.3, p.84-92, 2011.
RIZZON, L.A.; MIELE, A. Avaliação da cv. Cabernet Sauvignon para elaboração de vinho tinto. Ciência e Tecnologia de Alimentos, Campinas, v. 22, n.2, p.192-198, 2002.

SANTOS, C.E.; ROBERTO, S.R.; SATO, A.F.; JUBILEU, B.S.; AGUIAR, R.S. Características físicas, químicas e produtivas das videiras 'Cabernet Sauvignon' e 'Tannat' na região norte do Paraná. Acta Scientiarum Agronomy, Maringá, v.29, p.623629, 2007. Suplemento

SANTOS, H.P.; AMARANTE, C.V.T.; STEFFENS, C.A.; VENTURA, D.W.; MIQUELOTO, A. Qualidade da uva 'Cabernet Sauvignon' submetida ao raleio de cachos no sistema de condução latada. Revista de Ciências Agroveterinárias, Lages, v.9, n.2, p.160-168, 2010.

SCHUCK, E.; CALIARI, V.; ROSIER, J.P. Uso da plasticultura na melhoria da qualidade de frutas. In: ENFRUTE, 7., 2004, Friburgo. Anais... 8p. 